\title{
Design and Analysis of a New Tool Holder with Force Sensing Element
}

\author{
Zhengyou Xie \\ School of Mechatronics Engineering \\ Harbin Institute of Technology \\ Harbin, China \\ xiezhengyou@126.com \\ Yong Lu \\ School of Mechatronics Engineering \\ Harbin Institute of Technology \\ Harbin, China \\ luyong@hit.edu.cn
}

\author{
Jianguang Li \\ School of Mechatronics Engineering \\ Harbin Institute of Technology \\ Harbin, China \\ mejgli@hit.edu.cn
}

\begin{abstract}
Cutting force measurement is an essential requirement in the machining process. Hence, various methods of measuring the cutting force have been proposed by many researchers. In this study, a new type of tool holder with force sensing element is designed to measure the threedimensional cutting force and torque. A standard CNC tool holder is modified to produce deformable beams which create deformation concentration under the action of cutting force, and the tiny deformations in the proper positions of the beams could be used to calculate the cutting force and torque. Theoretical analysis of force model has been done. Then, the parameters of force sensing element have been optimized by using ANSYS Workbench to maximize sensitivities and minimize the negative influence on tool holder's stiffness and natural frequency. Results of the tests showed the new structure has achieved the performance as designed with good structural stiffness, low cross interference, high sensitivities and natural frequency.
\end{abstract}

Keywords-Cutting Force Measurement; Force Sensing Element; Deformable Beam; Force Model; Structure Optimization

\section{INTRODUCTION}

Modern machining requires faster cutting speed and higher machining precision, so the cutting process monitoring becomes particularly important. The cutting force is one of the most important machining process variables, and it provides significant information for tool wear monitoring, cutting process optimization, surface roughness prediction and others [1-4]. Thus, various methods to measure cutting force in turning, milling and drilling have been proposed by many researchers.

Table dynamometers based on piezoelectric effect are most widely used in laboratories for fundamental research at present, since they can provide highly accurate measurement of cutting forces. When using, a workpiece is mounted on top of the dynamometer and the dynamometer is clamped to the machine table, which undoubtedly limits the geometry and dimension of the workpiece. Coupled with table dynamometers' complicated installation and high cost, they are not suitable instruments for industrial use [1, 5, and 6].

Nowadays, flexibility and reconfigurability are the most significant requirements for cutting force measurement, which means the sensor system must have a sufficiently broad operating range to allow for various cutting tool sizes and workpiece configurations. Therefore, there has been interest in developing a rotating force sensing system built into the tool holder or machine tool. A spindle-integrated force sensor using a piezoelectric ring has been proposed for milling and drilling processes by Park et al. [8], Byrne and O'Donnell [9]. Totis et al. [10] developed a rotating dynamometer using $3 \mathrm{D}$ piezoelectric for measuring triaxial cutting force components in face milling, and the sensors were clamped in the tool holder. Yuxiang Liu [11] developed sensor vector on tool holder to detect cutting force using piezoelectric transducer. However, it is difficult to use piezoelectric sensor to measure static forces over a long period without drift due to the principle of piezoelectric sensor itself. Alternatively, forces can be estimated from the elastic deformation that can be measured by capacitive sensor, strain gauge et al. Albrecht et al. [12] integrated the capacitive sensors into spindle to indirectly measure radial force in milling process. Rizal [5] designed a force sensing element on which strain gauges were mounted and then integrated it on a tool holder to measure cutting force. Similarly to Suprock [6] and Nichols [7], who designed a sensorintegrated tool holder for torque measurement, which was called smart tool holder in their studies.

Structure design and analysis of force sensing element are required inevitably when adopting the sensorintegrated methods to measure cutting force, and many researchers have proposed various structure types: symmetrical cross beam type [5], thin-walled cylindrical structure [13] and octagonal ring structure [14, 15] et al. For a sensor-integrated system to be accepted by industry, the following criteria must be met $[5,6,16]$ :

Simple structure.

Compatible with existing tool types.

Easy to install, replace and reconfigure. 
High structural stiffness and sensitivity.

High natural frequency.

Low cross interference.

This present study makes a further contribution in addressing the issues, dealing with the design and construction of a new force sensing element on a tool holder. A standard CNC tool holder is modified to produce deformable beams structure. Then theoretical analysis of force model has been done, and the structure of force sensing element is optimized by using ANSYS Workbench Results of the tests showed the new structure has good structural stiffness, low cross interference, high sensitivities and natural frequency.

\section{Design AND Model of Force Sensing Element}

The cutting force is complicated and time-varying when the milling cutter cuts the workpiece material. However, for the convenience of functional analysis, the cutting force is decomposed into three dimensional orthogonal forces ( Fx, Fy and Fz) and torque (T) at a certain time as illustrated in Figure 1(a).

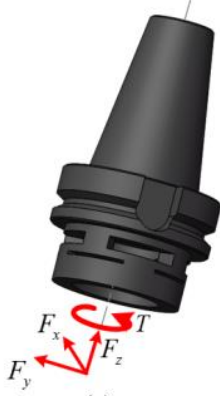

(a)

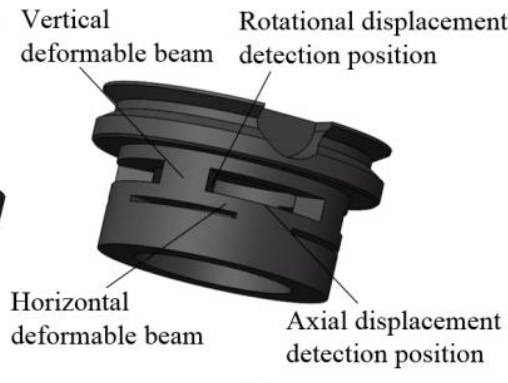

(b)
Figure 1. Model of force sensing element: (a) Perspective view (b) Enlarged view

The objective of this study is to design a new type of force sensing element on tool holder which can detect these forces and torque. Figure 1(b) illustrates the geometrical design of the force sensing element. Horizontal deformable beams and vertical deformable beams which can make deformation concentrated are created by making grooves in a standard $\mathrm{CNC}$ tool holder. The deformations in the proper positions of beams can be detected by capacitive sensor. Then, the cutting forces and torque can be calculated by these deformations.

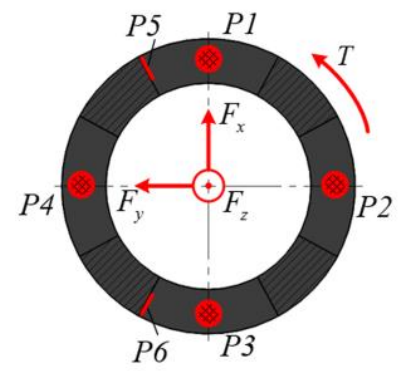

Figure 2. Displacement detection position

Considering the decoupling needs for three forces and torque, 6 displacement detection positions (P1 P6) are set on the beams as shown in Figure 2. Each force and torque can be solved out based on 6 sets of deformation data by using (1) due to the structure symmetry.

$$
\left\{\begin{array}{l}
F_{z}=k_{1} \frac{\Delta d_{1}+\Delta d_{2}+\Delta d_{3}+\Delta d_{4}}{4} \\
F_{x}=k_{2} \frac{\Delta d_{1}-\Delta d_{3}}{2} \\
F_{y}=k_{3} \frac{\Delta d_{4}-\Delta d_{2}}{2} \\
T=k_{4} \frac{\Delta d_{5}-\Delta d_{6}}{2}
\end{array}\right.
$$

Where $\Delta d_{i}(\mathrm{i}=1 \sim 6)$ is the deformation data of each position ( $\mathrm{Pi}(\mathrm{i}=1 \sim 6))$, $\mathrm{ki} \quad(\mathrm{i}=1 \sim 6)$ is certain coefficient related to structural stiffness which can be determined by experiment.

\section{FORCE MODEL}

Force model provides significant information to understand which parameters influence deformation and structural stiffness most, and makes it easier to complete structure optimization. The geometry of the force sensing element in this study includes 4 horizontal beams and 4 vertical deformable beams as shown in Figure 3.

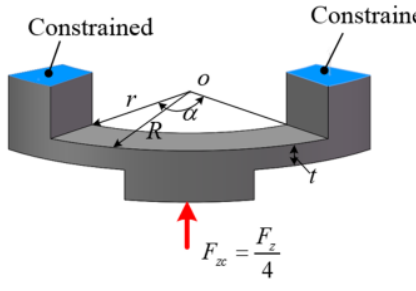

(a)

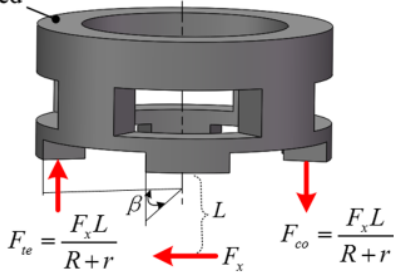

(b)

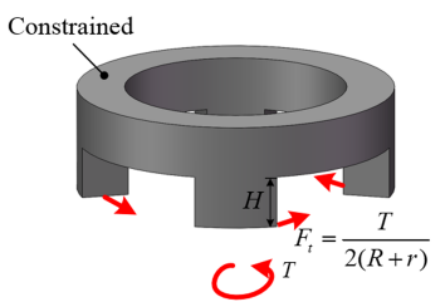

(c)

Figure 3. Force action on the beams model: (a) Axial force action (b) Radial force action (c) Torque action

Under the action of axial force (Fz), the force model is simplified to a simply supported beam considering the structure symmetry and in the center of which a force $\left(F_{z c}=F_{z} / 4\right.$ ) acts as shown in Figure 3(a). The force (Fzc) can be regarded as concentrated load if the central angle of the groove underneath $(\beta)$ is big enough. Then, the maximum deformation ( $\Delta d_{z z}$ ) caused by the axial force (Fz) can be obtained as

$$
\Delta d_{z z}=\frac{F_{z c} l_{H}^{3}}{48 E I_{H}^{3}}
$$

Where $1 \mathrm{H}$ is the length of the horizontal beam $\left(l_{H}=(R+r) \alpha / 2\right), \mathrm{R}$ is the outer radius, $\mathrm{r}$ is the inner radius, $\alpha$ is the central angle of the beam, $t$ is the thickness of horizontal beam, $\mathrm{E}$ is the modulus of elasticity and $\mathrm{IH}$ is 
the inertia moment of horizontal beam $\left(I_{H}=(R-r) t^{3} / 12\right)$. By substituting $\mathrm{IH}, \mathrm{IH}$ and Fzc into (2), axial sensitivity can be obtained as

$$
\frac{\Delta d_{z z}}{F_{z}}=\frac{(R+r)^{3} \alpha^{3}}{128 E(R-r) t^{3}}
$$

The axial deformation in the center of each horizontal beam caused by radial force (Fx or Fy), take Fx for instance, on the milling cutter is approximately equal to that caused by bending moment (FxL) on sensing element in Figure 3(b). The bending moment created the phenomenon that compressive force (Fte) acts on one side and tensile force (Fco) on the other. Because of the symmetry, the magnitudes of two deformations are equivalent while the directions are opposite. Hence, radial sensitivity can be written as (4) by changing Fzc to Fte (or Fco) in (2).

$$
\frac{\Delta d_{x z}}{F_{x}}=\frac{L(R+r)^{2} \alpha^{3}}{32 E(R-r) t^{3}}
$$

Where $\Delta d_{x z}$ is the maximum deformation of the horizontal beam caused by radial force $(\mathrm{Fx})$.

Under the action of torque ( $\mathrm{T})$, the force model is simplified to the cantilever beams and a tangential force ${ }_{(} F_{t}=T / 2(R+r)$ ) acts at theend of each beam as shown in Figure 3(c). The maximum deformation of vertical beam caused by the torque $(T)$ can be written as

$$
\Delta d_{T}=\frac{F_{t} H^{3}}{12 E I_{V}}
$$

Where $I_{V}$ is the inertia moment of vertical beam $\left(I_{V}=(R-r)(\pi / 2-\alpha)^{3}(R+r)^{3} / 96\right)$. By substituting $F_{t}$, and $I_{V}$ into (5), torsional sensitivity can be obtained as

$$
\frac{\Delta d_{T}}{T}=\frac{8 H^{3}}{E(R-r)(\pi-2 \alpha)^{3}(R+r)^{3}}
$$

\section{FinITE ELEMENT ANALYSIS}

In order to understand the deformation distribution of the force sensing element under each force and to determine optimal dimensions of structure, ANSYS Workbench has been used to perform the static analysis and modal analysis.

The three-dimensional model of the modified tool holder with force sensing element was established based on the prototype of BT50SLN40-150 and entered into ANSYS Workbench to do the pre-treatment of FEA. Considering the real constraint and load conditions, the taper shank of tool holder was constrained and the each force (axial force, radial force and torque) individually acted at the tool holder. Then, the deformation nephograms of beams under each force were achieved as shown in Figure 4. Figure 4(a) shows all the 4 horizontal beams' deformations have the same magnitude and direction under the action of axial force. Moreover, under the action of radial force, the deformations of two horizontal beams along the force direction are equivalent while the directions are opposite, and other two horizontal beams' deformations are near to zero as shown in Figure 4(b). Figure 4(c) shows the deformation distribution of vertical beam under the action of torque. All the analysis results fit the force model well.

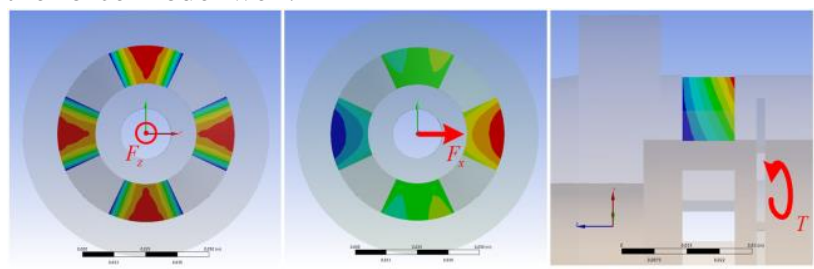

(a)

(b)

(c)

Figure 4. Deformation nephograms under (a) axial force (b) radial force and (c) torque

The analysis of force model shows that outer radius ( $R$ ), inner radius $(r)$, thickness of horizontal beam $(t)$, length of vertical beam $(\mathrm{H})$ and central angle of the horizontal beam $(\alpha)$ influence the structural sensitivities most. However, the parameter $\beta$ was neglected in theoretical analysis, and the horizontal beams are acted upon by the uniform load but not a concentrated load if taking parameter $\beta$ into consideration. Thus, all these 6 parameters were regarded as design parameters when using ANSYS Workbench to optimize the structure, and the local sensitivity histograms of each design parameter on sensitivities and natural frequency are obtained in ANSYS as shown in Figure 5.

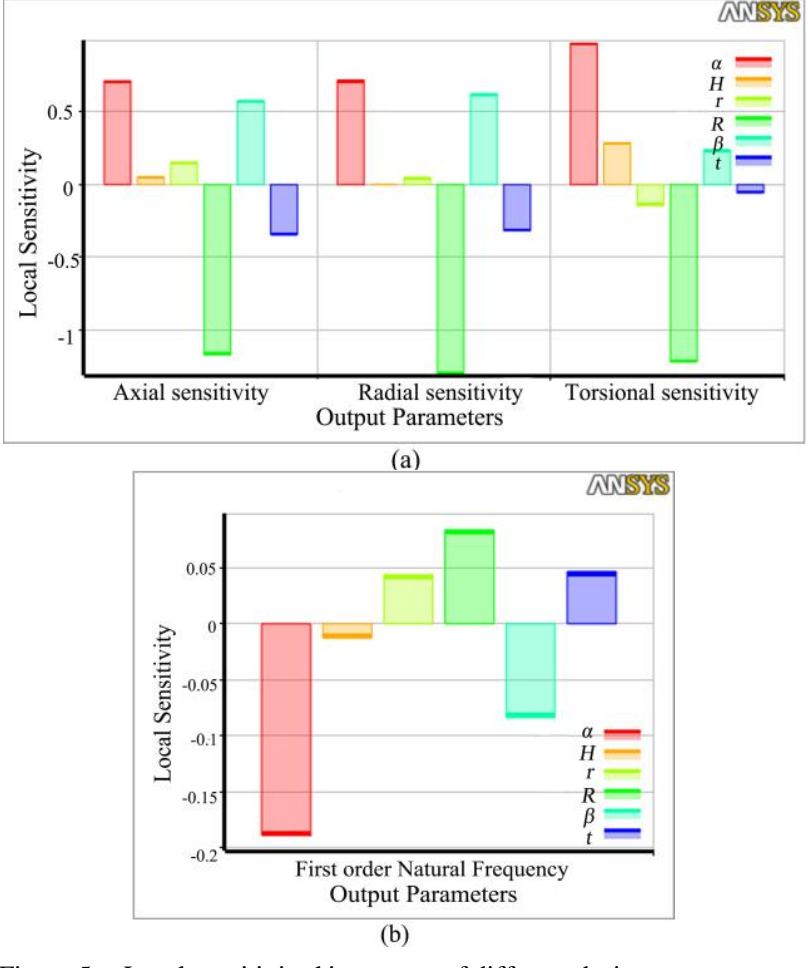

Figure 5. Local sensitivity histograms of different design parameters on (a) structural sensitivities and (b) fisrt order natural frequency

Figure 5 presents important information that the effect extent of any one of these design parameters $(\alpha, \mathrm{H}, \mathrm{r}, \mathrm{R}, \beta$, t) on different output parameters (sensitivities and natural frequency) For instance, Figure 5(a) shows that the length 
of vertical beam $(\mathrm{H})$ has little impact on both axial and radial sensitivities but by contrast, affects torsional sensitivity greatly that conforms to the force model well. The central angle of the horizontal beam $(\alpha)$ is closely related to the length of horizontal beams and the thickness of vertical beams, thus it impacts the sensitivities of all directions.

Figure 5(b) shows there is a negative correlation between the first order natural frequency and the two central angles of the horizontal beam and the groove underneath ( $\alpha$ and $\beta$ ), which means the bigger the $\alpha$ and $\beta$ are, the lower the natural frequency is, which is bad for the dynamic performance of tool holder. Moreover, there is a positive correlation between natural frequency and the outer radius (R), inner radius ( $r$ ) and thickness of horizontal beam (t). Thus, the dynamic performance of tool holder can be improved through increasing the magnitudes of $\mathrm{R}, \mathrm{r}$ and $\mathrm{t}$.

\section{CONSTRUCTION AND TEST}

Considering the balance of structural sensitivities, stiffness and natural frequency of tool holder, the dimensions of the structure were determined by using Goal Driven Optimization module in ANSYS Workbench. Wherein, $\mathrm{R}=80 \mathrm{~mm}, \mathrm{r}=40 \mathrm{~mm}, \mathrm{t}=4.5 \mathrm{~mm}, \mathrm{H}=12 \mathrm{~mm}, \alpha=50^{\circ}$ and $\beta=60^{\circ}$.The prototype of tool holder is BT50SLN40150 , and the material is $40 \mathrm{Cr}$

The experimental system was set up based on X62W horizontal milling machine to do a series of static tests on modified tool holder. Figure 6 shows the axial force load test system. Move the machine table slowly at regular intervals to load axial force and the magnitude of the force was detected by Kistler 9257B dynamometer. To achieve the deformations of 6 positions, 6 capacitive sensors were integrated in the tool holder. Each capacitive sensor had two plates, one was the moveable plate which was bonded to the measurement position and the other plate was fixed. When the beams deformed under the force, the moveable plate moved along with it and the plates' distance also changed, which caused the change of sensors' output voltage, then the deformations can be calculated.

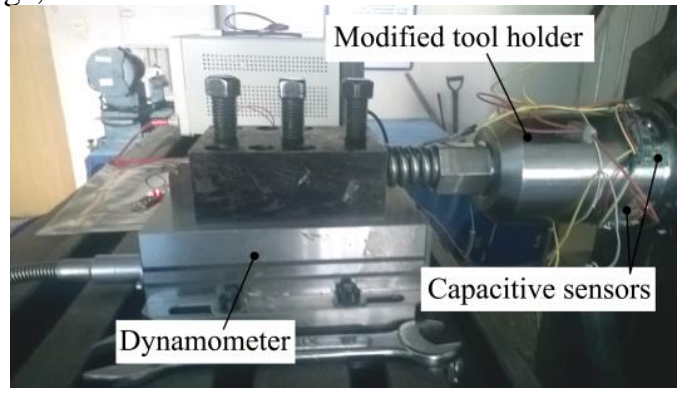

Figure 6. Axial force load test system

Figure 7 shows the deformation curve under the axial force. 4 horizontal beams deformed under the action of axial force, and the plates' distance decreased, which caused the sensors' output voltages increased, thus the calculated deformation values $\left(\Delta d_{1}, \Delta d_{2}, \Delta d_{3}\right.$ and $\left.\Delta d_{4}\right)$ were positive. However, because the tool holder is not symmetrical in the strict sense, nor are the 6 deformation detection positions and these 4 deformations of horizontal beams were not equal in magnitude as theoretical analysis and simulation analysis. Among all, the sensitivity of position $\mathrm{P} 2$ is maximum at $5.23 \times 10^{-4} \mu \mathrm{m} / \mathrm{N}$. While the 2 deformation values ( $\Delta d_{5}$ and $\Delta d_{6}$ ) of vertical beams were very small and negative. The reason is that each vertical beam was compressed under the axial force, which caused the position of the moveable plate changed and the effective overlap area of two capacitor plates reduced, thus the output voltages of these 2 capacitive sensors decreased and the calculated deformation values ( $\Delta d_{5}$ and $\left.\Delta d_{6}\right)$ were negative. Nonetheless, this didn't affect the interpretation of force sensing element's structural properties.

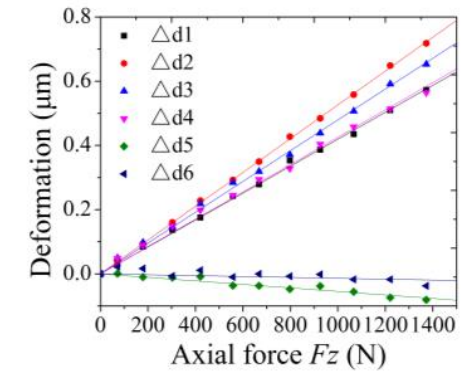

Figure 7. Deformation curve under the $F_{z}$

Moreover, the 6 sets of deformations of beams should be rearranged into 4 sets of deformations as described in (1), which means the relationship curves of $F_{z} \sim\left(\Delta d_{1}+\Delta d_{2}+\Delta d_{3}+\Delta d_{4}\right) / 4 \quad, \quad F_{x} \sim\left(\Delta d_{1}-\Delta d_{3}\right) / 2$, $F_{y} \sim\left(\Delta d_{4}-\Delta d_{2}\right) / 2$ and $T \sim\left(\Delta d_{5}-\Delta d_{6}\right) / 2$ are wanted.

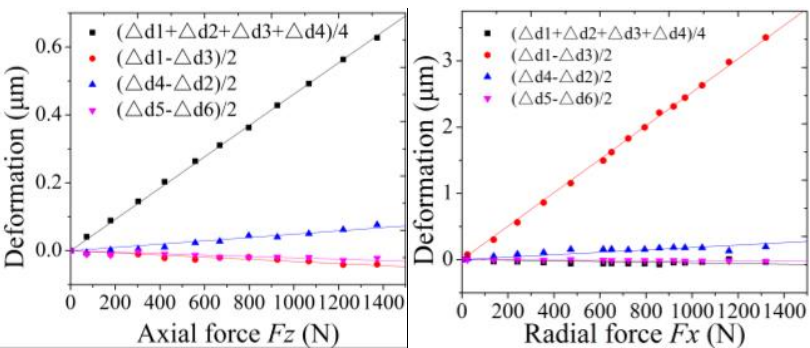
(a)

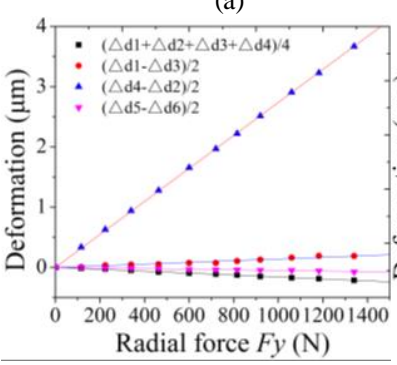

(c)

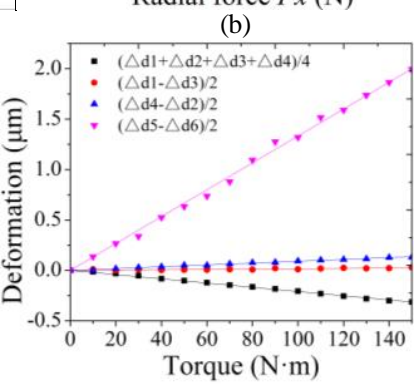

(d)
Figure 8. Rearranged curves under (a) axial force (b) radial force $F_{x}$ (c) radial force $F_{y}(\mathrm{~d})$ torque

Figure 8 shows the relationship curves after rearranged, which provides important quantitative information of force sensing element. Take Figure 8(a) for instance, under the action of axial force, the value of rearranged deformation $\left(\Delta d_{1}+\Delta d_{2}+\Delta d_{3}+\Delta d_{4}\right) / 4$ changed significantly while the other three changed little, which is advantageous to the cutting force measurement system. But due to the asymmetry of structure and detection positions, the magnitudes of the 4 deformations ( $\Delta d_{1}, \Delta d_{2}, \Delta d_{3}$ and $\Delta d_{4}$ ) 
are not equal, thus $\left(\Delta d_{1}-\Delta d_{3}\right) / 2$ and $\left(\Delta d_{4}-\Delta d_{2}\right) / 2$ are not zero, and $\left(\Delta d_{5}-\Delta d_{6}\right) / 2$ is not zero either. Similarly, the other 3 curves in Figure 8 showed the phenomenon also appeared under Fx, Fy and T, which is known as cross interference. And it is usually impossible to be eliminated because that the tool holder was developed by a single force sensing element to measure three perpendicular cutting force components and torque simultaneously. The cross interference of this system is small. However, an effective decoupling algorithm is required in practice.

The structural stiffness of tool holder is weakened after modified to produce deformable beams. Detailed data are reported in Table 1.

Compared the data before and after modified, the stiffness of tool holder in every direction has decreased around $50 \%$, while still meets requirements recommended by CIRP.

TABLE I. STRUCTURAL STIFFNESS

\begin{tabular}{|c|c|c|c|}
\hline & Before modified & After modified & Decrease \\
\hline $\begin{array}{c}\text { Axial } \\
\text { direction }\end{array}$ & $4.224 \times 10^{9} \mathrm{~N} / \mathrm{m}$ & $1.731 \times 10^{9} \mathrm{~N} / \mathrm{m}$ & $58.8 \%$ \\
\hline $\begin{array}{c}\text { Radial } \\
\text { direction }\end{array}$ & $2.647 \times 10^{8} \mathrm{~N} / \mathrm{m}$ & $1.206 \times 10^{8} \mathrm{~N} / \mathrm{m}$ & $54.4 \%$ \\
\hline $\begin{array}{c}\text { Torsional } \\
\text { direction }\end{array}$ & $3.897 \times 10^{6} \mathrm{~N} \cdot \mathrm{m} / \mathrm{rad}$ & $2.131 \times 10^{6} \mathrm{~N} \cdot \mathrm{m} / \mathrm{rad}$ & $45.2 \%$ \\
\hline
\end{tabular}

The first order natural frequency of the modified tool holder is also determined after test, which is $1719.1 \mathrm{~Hz}$ and meets the requirement of normal use.

\section{CONCLUSION}

In this work, a new tool holder with force sensing element which can be used to measure three perpendicular cutting force components and torque was developed. Force model was established to determine the key structural parameters which influence sensitivities most. Then, considering the needs of structural sensitivities, stiffness and natural frequency, the structure was optimized by using ANSYS Workbench. A series of tests showed that the modified tool holder has good structural stiffness, low cross interference, high sensitivities and natural frequency.

\section{ACKNOWLEDGMENT}

This research was supported by National High Technology Research and Development Program of China (863 Program) under Grant 2013AA041107.

\section{REFERENCES}

[1] G. Byrne, D. Dornfeld, I. Inasaki, G. Ketteler, W. K Nig, and R. Teti, "Tool Condition Monitoring (TCM) - The Status of
Research and Industrial Application," CIRP Annals Manufacturing Technology, vol. 44, pp. 541-567, 1995.

[2] M. Nouri, B. K. Fussell, B. L. Ziniti, and E. Linder, "Real-time tool wear monitoring in milling using a cutting condition independent method," International Journal of Machine Tools and Manufacture, vol. 89, pp. 1-13, 2015.

[3] C. H. Lauro, L. C. Brandão, D. Baldo, R. A. Reis, and J. P. Davim, "Monitoring and processing signal applied in machining processes - A review," Measurement, vol. 58, pp. 73-86, 2014.

[4] R. Teti, K. Jemielniak, G. O Donnell, and D. Dornfeld, "Advanced monitoring of machining operations," CIRP Annals Manufacturing Technology, vol. 59, pp. 717-739, 2010.

[5] M. Rizal, J. A. Ghani, M. Z. Nuawi, and C. H. Che Haron, "Development and testing of an integrated rotating dynamometer on tool holder for milling process," Mechanical Systems and Signal Processing, vol. 52-53, pp. 559 - 576, 2015.

[6] C. A. Suprock and J. S. Nichols, "A low cost wireless high bandwidth transmitter for sensor-integrated metal cutting tools and process monitoring," International Journal of Mechatronics and Manufacturing Systems, vol. 2, pp. 441-454, 2009.

[7] J. S. Nichols, "Design and application of a wireless torque sensor for CNC milling," vol. Master of Science: ProQuest, UMI Dissertations Publishing, 2009. p. 112.

[8] S.S. Park, "High Frequency Bandwidth Cutting Force Measurements in Milling Using the Spindle Force Sensor System," vol. Doctorate: University of British Columbia, Vancouver, Canada, 2004.

[9] G. Byrne and G. E. O Donnell, "An Integrated Force Sensor Solution for Process Monitoring of Drilling Operations," CIRP Annals - Manufacturing Technology, vol. 56, pp. 89-92, 2007.

[10] G. Totis, G. Wirtz, M. Sortino, D. Veselovac, E. Kuljanic, and F. Klocke, "Development of a dynamometer for measuring individual cutting edge forces in face milling," Mechanical Systems and Signal Processing, vol. 24, pp. 1844-1857, 2010.

[11] Yuxiang Liu, " The Design of Sensor on Cutting Force Measurement System," vol. Master: Shenyang Ligong University, 2012, p. 52.

[12] A. Albrecht, S. S. Park, Y. Altintas, and G. Pritschow, "High frequency bandwidth cutting force measurement in milling using capacitance displacement sensors," International Journal of Machine Tools and Manufacture, vol. 45, pp. 993-1008, 2005.

[13] G. Dini and F. Tognazzi, "Tool condition monitoring in end milling using a torque-based sensorized toolholder," Proceedings of the Institution of Mechanical Engineers, Part B: Journal of Engineering Manufacture, vol. 221, pp. 11-23, 2007.

[14] S. Karabay, "Analysis of drill dynamometer with octagonal ring type transducers for monitoring of cutting forces in drilling and allied process," Materials \& Design, vol. 28, pp. 673-685, 2007.

[15] S. Yaldız, F. Ünsaçar, H. Sağlam, and H. Işık, "Design, development and testing of a four-component milling dynamometer for the measurement of cutting force and torque," Mechanical Systems and Signal Processing, vol. 21, pp. 1499-1511, 2007.

[16] Pengfei Kuang, Fan Wang, Xinyong Li and Miaomiao Tian, "Structure Design of Sensor Vector Tool Holder," New Technology \& New Process, pp. 66-68, 2013. 